\title{
Hurricane Katrina: Influence on the Male-to-Female Birth Ratio
}

\author{
Victor Grech ${ }^{\mathrm{a}}$ Hagen Scherb ${ }^{\mathrm{b}}$

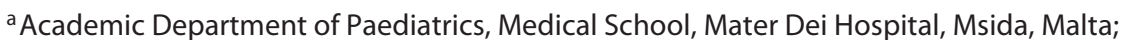 \\ ${ }^{\mathrm{b}}$ Institute of Computational Biology, Helmholtz Zentrum München, Neuherberg, Germany
}

\author{
Key Words \\ Birth rate $\cdot$ Cyclonic storms - Sex ratio - Disasters - Florida \\ Alabama $\cdot$ Louisiana $\cdot$ Mississippi
}

\begin{abstract}
Objective: This study was carried out in order to ascertain whether or not Hurricane Katrina and related factors (i.e. the amount of rainfall) influenced the male-to-female birth ratio (M/F). Materials and Methods: Monthly births by gender for the affected states (Alabama, Florida, Louisiana and Mississippi) for January 2003 to December 2012 were obtained from the Centers for Disease Control and Prevention (CDC Wonder, Atlanta, Ga., USA). Precipitation data was obtained from the US National Weather Service. Ordinary linear logistic regression was used for trend analysis. A $p$ value $\leq 0.05$ was taken to represent a statistically significant result. $\boldsymbol{R} \boldsymbol{e}$ sults: Of the total of 3,903,660 live births, 1,996,966 (51.16\%) were male and 1,906,694 (48.84\%) were female. Significant seasonal variation was noted (the maximum M/F in May was 1.055 , the minimum M/F in September was 1.041, $p=0.0073$ ). There was also a separate and significant rise in M/F 8-10 months after the storm (April to June 2006, peak M/F 1.078, $p=0.0074)$, which translated to an approximate deficit of 800 girls compared to 46,072 girls born in that period if the $\mathrm{M} / \mathrm{F}$ increase was theoretically only due to a girls' deficit in the denominator of the ratio. This spike was only present in
\end{abstract}

Alabama, Louisiana and Mississippi, all of which received heavy rainfall. Florida did not receive heavy rainfall and experienced no such M/F spike. Conclusion: In this study there was a dose-response relation between the amount of rainfall after Hurricane Katrina and the monthly sex ratio of live births in the US states of Alabama, Louisiana and Mississippi 8-10 months later. The well-known yet unexplained distinct sex ratio seasonality may be due to natural or man-made radiation contained in the rain.

(c) 2015 S. Karger AG, Basel

\section{Introduction}

In humans, live male births exceed live female births by approximately $4-6 \%$ [1]. This ratio is conventionally expressed as $\mathrm{M} / \mathrm{F}$, the sex ratio, or sex odds. A multitude of factors have been implicated as influencing this ratio and most appear to reduce it by inducing spontaneous termination of pregnancy, which affects more male than female foetuses $[1,2]$. Other factors include natural disasters such as floods [3] and earthquakes [4], as well as events precipitated by man, including short wars [5], the Great London Smog [3] and terrorist events [6].

Hurricane Katrina formed over the Bahamas in August 2005 and wrought havoc over the southern USA. The storm traversed Florida as a category 1 hurricane $(\leq 74$

\begin{tabular}{ll}
\hline KARGER 125.3 & $\begin{array}{l}\text { (2) 2015 S. Karger AG, Basel } \\
1011-7571 / 15 / 0245-0477 \$ 39.50 / 0 \quad \text { Karger }\end{array}$ \\
$\begin{array}{l}\text { E-Mail karger@karger.com } \\
\text { www.karger.com/mpp }\end{array}$ & $\begin{array}{l}\text { This is an Open Access article licensed under the terms of the } \\
\text { Creative Commons Attribution-NonCommercial 3.0 Un- } \\
\text { ported license (CC BY-NC) (www.karger.com/OA-license), } \\
\text { applicable to the online version of the article only. Distribu- } \\
\text { tion permitted for non-commercial purposes only. }\end{array}$
\end{tabular}

Prof. Victor Grech

Department of Paediatrics

Mater Dei Hospital

MT-2090 Msida (Malta)

E-Mail victor.e.grech@gov.mt 
Fig. 1. Precipitation maps of the USA (grey shading) from August 29 to September 1, 2005 (source: US National Weather Service, see table 2) (colour online version only).

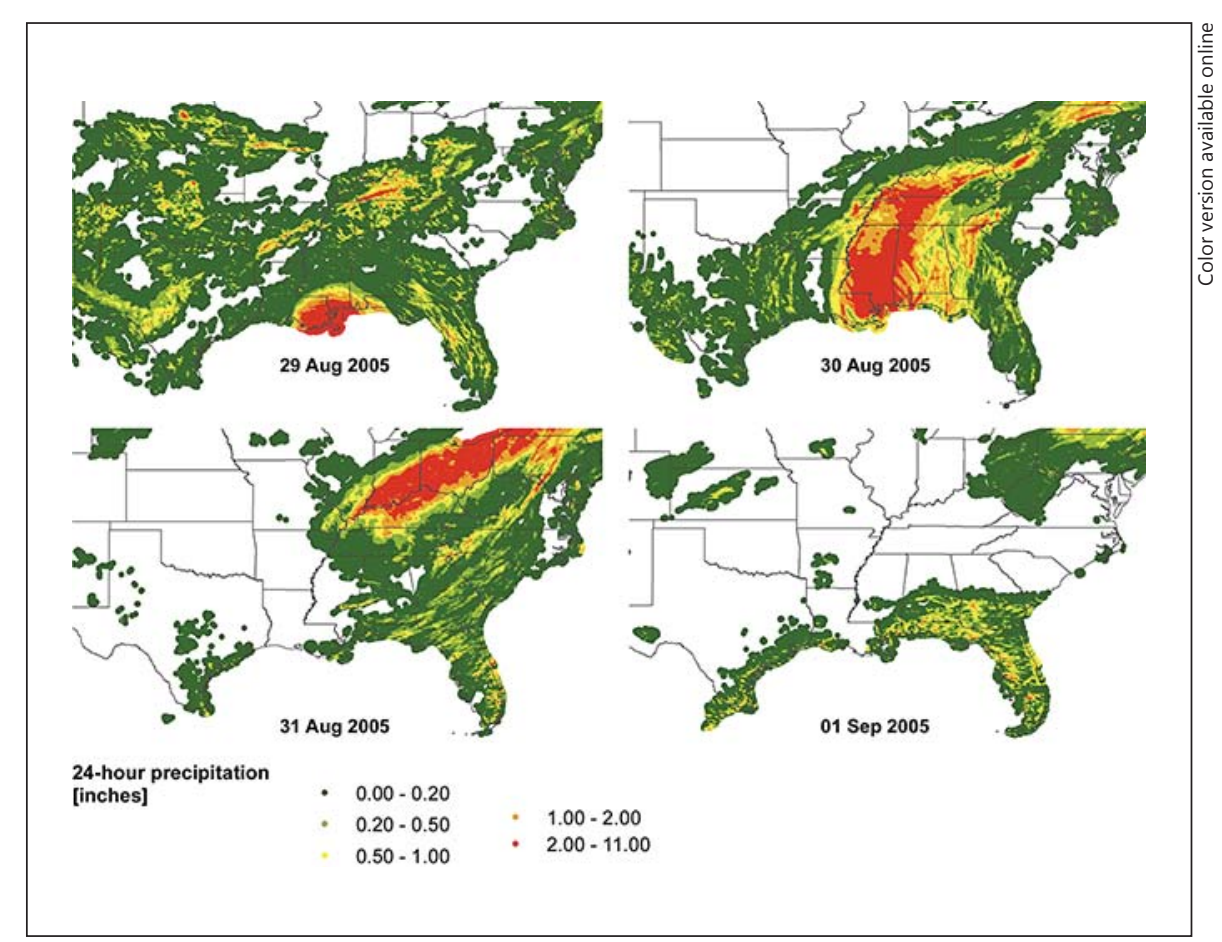

$\mathrm{mph})$. It then strengthened over the Gulf of Mexico and struck southeast Louisiana as a category 3 event $(\leq 111$ $\mathrm{mph}$ ) as well as Mississippi, waning away over Alabama $[7,8]$.

The associated storm surge caused widespread destruction along the Gulf coast and severe floods in New Orleans, which lingered for several weeks. The surge protection failures were classified as the worst civil engineering disaster in the history of the USA $[7,8]$.

It is known that calamitous events promote spontaneous abortions in stressed, pregnant women [6]. This was evidenced in the USA after the attacks of September 11, 2001. Such losses are male-biased, skewing the sex ratio at birth toward female babies [9]. After Hurricane Katrina, a foetal loss phenomenon was noted (gender unspecified), and this was even related in degree to the extent of property damage [10].

It is also known that $\mathrm{M} / \mathrm{F}$ is subject to seasonality [11], and that there is a positive association with rainfall levels 9-11 months before birth [12]. Hurricane Katrina was accompanied by heavy rainfall. It brought more rain to Alabama, Louisiana and Mississippi than to Florida because the storm only passed over the northwest tip of Florida $[7,8]$.

This study was carried out in order to ascertain whether or not this natural event influenced M/F in Alabama,
Florida, Louisiana and Mississippi. It was also attempted to determine whether or not the disparate rainfall in these states had a differential effect on M/F.

\section{Subjects and Methods}

Monthly male and female live births by state for Alabama, Florida, Louisiana and Mississippi in the USA were obtained from the website of the Centers for Disease Control and Prevention (CDC), as shown in table 1 [13]. The time period analysed was January 2003 to December 2012.

Precipitation data for Alabama, Florida, Louisiana and Mississippi were obtained from the US National Weather Service (table 2) [14]. Precipitation maps of the USA from August 29 to September 1, 2005, are presented in figure 1. Ordinary linear logistic regression was used in order to assess time trends in the occurrence of male live births and to investigate whether there were changes in the trend functions after distinct events. This involved considering the male proportion among all male $(\mathrm{m})$ and female $(\mathrm{f})$ births: $\mathrm{p}_{\mathrm{m}}=\mathrm{m} /(\mathrm{m}+\mathrm{f})$. Important and useful parameters in this context are the sex odds, $S O=\mathrm{p}_{\mathrm{m}} /\left(1-\mathrm{p}_{\mathrm{m}}\right)=\mathrm{m} / \mathrm{f}$, and the sex odds ratio (SOR), which is the ratio of two sex odds of interest, i.e. in exposed versus non-exposed populations. Dummy coding was used for single points in time and for time periods. For example, the dummy variable for the time window from 2006 onwards was defined as $\mathrm{d}_{2006}(\mathrm{t})=0$ for $\mathrm{t}<2006$, and $\mathrm{d}_{2006}(\mathrm{t})=1$ for $\mathrm{t} \geq 2006$. The SOR peak is defined as the expected sex odds in the time interval from April to June 2006 (4-6/2006) divided by the expected baseline sex odds in that same time interval. The simple and parsimonious lo- 
Table 1. Monthly live births for Alabama, Florida, Louisiana and Mississippi, 2003-2012

\begin{tabular}{|c|c|c|c|c|c|c|c|c|c|}
\hline \multicolumn{2}{|l|}{ Date } & \multicolumn{2}{|c|}{ Alabama } & \multicolumn{2}{|l|}{ Florida } & \multicolumn{2}{|c|}{ Louisiana } & \multicolumn{2}{|c|}{ Mississippi } \\
\hline year & month & female & male & female & male & female & male & female & male \\
\hline \multirow[t]{12}{*}{2003} & Jan & 2,318 & 2,548 & 8,546 & 8,970 & 2,626 & 2,781 & 1,685 & 1,834 \\
\hline & Feb & 2,150 & 2,287 & 7,806 & 8,322 & 2,364 & 2,486 & 1,535 & 1,607 \\
\hline & Mar & 2,390 & 2,467 & 8,238 & 8,815 & 2,467 & 2,508 & 1,649 & 1,757 \\
\hline & Apr & 2,241 & 2,465 & 8,123 & 8,594 & 2,490 & 2,609 & 1,616 & 1,608 \\
\hline & May & 2,321 & 2,517 & 8,410 & 8,936 & 2,596 & 2,640 & 1,584 & 1,717 \\
\hline & Jun & 2,330 & 2,473 & 8,097 & 8,557 & 2,455 & 2,617 & 1,743 & 1,762 \\
\hline & Jul & 2,600 & 2,692 & 8,648 & 9,251 & 2,756 & 2,991 & 1,760 & 1,939 \\
\hline & Aug & 2,580 & 2,635 & 9,167 & 9,507 & 2,883 & 2,969 & 1,867 & 1,954 \\
\hline & Sep & 2,669 & 2,652 & 9,272 & 9,965 & 2,961 & 2,970 & 1,856 & 1,859 \\
\hline & Oct & 2,572 & 2,639 & 9,347 & 9,842 & 2,774 & 2,955 & 1,883 & 1,930 \\
\hline & Nov & 2,327 & 2,400 & 8,507 & 8,695 & 2,541 & 2,754 & 1,698 & 1,719 \\
\hline & Dec & 2,558 & 2,721 & 9,105 & 9,530 & 2,874 & 2,973 & 1,832 & 1,986 \\
\hline \multirow[t]{12}{*}{2004} & Jan & 2,326 & 2,490 & 8,621 & 9,186 & 2,763 & 2,714 & 1,672 & 1,736 \\
\hline & Feb & 2,188 & 2,289 & 8,174 & 8,446 & 2,381 & 2,450 & 1,626 & 1,591 \\
\hline & Mar & 2,336 & 2,571 & 8,682 & 8,964 & 2,642 & 2,739 & 1,751 & 1,834 \\
\hline & Apr & 2,311 & 2,358 & 8,496 & 8,869 & 2,477 & 2,673 & 1,679 & 1,724 \\
\hline & May & 2,340 & 2,431 & 8,292 & 8,878 & 2,377 & 2,536 & 1,639 & 1,688 \\
\hline & Jun & 2,386 & 2,552 & 8,640 & 9,065 & 2,667 & 2,785 & 1,737 & 1,780 \\
\hline & Jul & 2,461 & 2,671 & 9,112 & 9,488 & 2,721 & 2,910 & 1,810 & 1,863 \\
\hline & Aug & 2,491 & 2,640 & 9,136 & 9,731 & 2,730 & 2923 & 1,813 & 1,923 \\
\hline & Sep & 2,526 & 2,717 & 9,578 & 9,968 & 2,859 & 3,039 & 1,930 & 1,902 \\
\hline & Oct & 2,421 & 2,640 & 9,187 & 9,724 & 2,813 & 2,888 & 1,755 & 1,897 \\
\hline & Nov & 2,490 & 2,617 & 8,857 & 9,531 & 2,716 & 2,820 & 1,768 & 1,914 \\
\hline & Dec & 2,605 & 2,653 & 9,525 & 9,903 & 2,808 & 2,938 & 1,791 & 2,004 \\
\hline \multirow[t]{12}{*}{2005} & Jan & 2,386 & 2,570 & 9,160 & 9,317 & 2,578 & 2,783 & 1,743 & 1,792 \\
\hline & Feb & 2,175 & 2,303 & 8,278 & 8,710 & 2,386 & 2,480 & 1,506 & 1,634 \\
\hline & Mar & 2,447 & 2,506 & 9,019 & 9,500 & 2,618 & 2,767 & 1,629 & 1,846 \\
\hline & Apr & 2,331 & 2,346 & 8,517 & 8,756 & 2,424 & 2,468 & 1,570 & 1,670 \\
\hline & May & 2,429 & 2,476 & 8,698 & 9,232 & 2,553 & 2,647 & 1,648 & 1,826 \\
\hline & Jun & 2,533 & 2,644 & 8,987 & 9,425 & 2,582 & 2,817 & 1,794 & 1,745 \\
\hline & Jul & 2,499 & 2,609 & 9,224 & 9,743 & 2,548 & 2,561 & 1,766 & 1,794 \\
\hline & Aug & 2,613 & 2,808 & 9,949 & 10,433 & 2,551 & 2,691 & 1,862 & 1,895 \\
\hline & Sep & 2,636 & 2,715 & 10,189 & 10,454 & 2,523 & 2,663 & 1,851 & 1,911 \\
\hline & Oct & 2,443 & 2,600 & 9,416 & 9,928 & 2,357 & 2,409 & 1,760 & 1,851 \\
\hline & Nov & 2,578 & 2,550 & 9,717 & 9,872 & 2,314 & 2,366 & 1,745 & 1,778 \\
\hline & Dec & 2,543 & 2,713 & 9,574 & 10,142 & 2,346 & 2,505 & 1,848 & 1,931 \\
\hline \multirow[t]{12}{*}{2006} & Jan & 2,510 & 2,566 & 9,416 & 9,830 & 2,367 & 2,569 & 1,795 & 1,880 \\
\hline & Feb & 2,325 & 2,471 & 8,908 & 9,004 & 2,211 & 2,231 & 1,682 & 1,718 \\
\hline & Mar & 2,544 & 2,694 & 9,496 & 10,015 & 2,448 & 2,591 & 1,779 & 1,847 \\
\hline & Apr & 2,286 & 2,479 & 8,547 & 9,028 & 2,166 & 2,290 & 1,620 & 1,763 \\
\hline & May & 2,420 & 2,652 & 9,103 & 9,683 & 2,275 & 2,546 & 1,701 & 1,930 \\
\hline & Jun & 2,434 & 2,658 & 9,282 & 9,743 & 2,463 & 2,665 & 1,775 & 1,941 \\
\hline & Jul & 2,670 & 2,706 & 9,725 & 9,995 & 2,670 & 2,826 & 1,806 & 2,028 \\
\hline & Aug & 2,836 & 3,044 & 10,544 & 11,067 & 2,932 & 3,193 & 2,140 & 2,212 \\
\hline & Sep & 2,694 & 2,809 & 10,530 & 10,839 & 2,905 & 2,973 & 2,090 & 2,184 \\
\hline & Oct & 2,666 & 2,846 & 10,298 & 10,672 & 2,834 & 2,935 & 2,050 & 2,056 \\
\hline & Nov & 2,613 & 2,852 & 9,788 & 10,401 & 2,754 & 2,910 & 1,944 & 2,066 \\
\hline & Dec & 2,611 & 2,846 & 10,180 & 10,708 & 2,783 & 2,839 & 1,984 & 2,065 \\
\hline
\end{tabular}


Table 1 (continued)

\begin{tabular}{|c|c|c|c|c|c|c|c|c|c|}
\hline \multicolumn{2}{|l|}{ Date } & \multicolumn{2}{|c|}{ Alabama } & \multicolumn{2}{|l|}{ Florida } & \multicolumn{2}{|c|}{ Louisiana } & \multicolumn{2}{|c|}{ Mississippi } \\
\hline year & month & female & male & female & male & female & male & female & male \\
\hline \multirow[t]{12}{*}{2007} & Jan & 2,622 & 2,693 & 9,850 & 10,554 & 2,756 & 2,876 & 1,916 & 2,076 \\
\hline & Feb & 2,496 & 2,440 & 8,977 & 9,560 & 2,454 & 2,554 & 1,763 & 1,799 \\
\hline & Mar & 2,621 & 2,722 & 9,649 & 10,135 & 2,652 & 2,747 & 1,924 & 1,994 \\
\hline & Apr & 2,462 & 2,646 & 9,053 & 9,387 & 2,439 & 2,518 & 1,680 & 1,754 \\
\hline & May & 2,633 & 2,691 & 9,578 & 10,031 & 2,560 & 2,668 & 1,802 & 1,902 \\
\hline & Jun & 2,450 & 2,609 & 9,299 & 9,940 & 2,651 & 2,650 & 1,821 & 1,935 \\
\hline & Jul & 2,801 & 2,879 & 9,902 & 10,581 & 2,778 & 2,929 & 1,896 & 2,068 \\
\hline & Aug & 2,985 & 2,927 & 10,680 & 11,127 & 2,975 & 3,172 & 2,061 & 2,167 \\
\hline & Sep & 2,708 & 2,850 & 9,777 & 10,134 & 2,831 & 2,894 & 1,927 & 2,006 \\
\hline & Oct & 2,771 & 2,859 & 9,949 & 10,575 & 2,834 & 2,978 & 1,974 & 2,069 \\
\hline & Nov & 2,700 & 2,843 & 10,047 & 10,257 & 2,867 & 2,836 & 1,888 & 2,026 \\
\hline & Dec & 2,693 & 2,703 & 9,881 & 10,242 & 2,788 & 2,894 & 2,017 & 2,026 \\
\hline \multirow[t]{12}{*}{2008} & Jan & 2,781 & 2,778 & 10,067 & 10,394 & 2,817 & 2,993 & 1,959 & 1,978 \\
\hline & Feb & 2,505 & 2,645 & 9,057 & 9,656 & 2,507 & 2,570 & 1,774 & 1,807 \\
\hline & Mar & 2,625 & 2,599 & 9,303 & 9,784 & 2,603 & 2,666 & 1,705 & 1,829 \\
\hline & Apr & 2,600 & 2,697 & 8,890 & 9,565 & 2,490 & 2,625 & 1,727 & 1,731 \\
\hline & May & 2,529 & 2,561 & 8,923 & 9,740 & 2,373 & 2,620 & 1,701 & 1,823 \\
\hline & Jun & 2,507 & 2,679 & 8,864 & 9,295 & 2,408 & 2,643 & 1,798 & 1,872 \\
\hline & Jul & 2,769 & 2,805 & 9,829 & 10,174 & 2,886 & 2,977 & 1,957 & 2,049 \\
\hline & Aug & 2,915 & 2,995 & 9,958 & 10,287 & 2,791 & 2,892 & 1,930 & 2,050 \\
\hline & Sep & 2,620 & 2,912 & 9,746 & 10,379 & 2,862 & 2,966 & 1,977 & 1,975 \\
\hline & Oct & 2,693 & 2,789 & 9,675 & 10,108 & 2,772 & 2,808 & 1,839 & 1,961 \\
\hline & Nov & 2,312 & 2,562 & 8,745 & 9,198 & 2,566 & 2,607 & 1,802 & 1,765 \\
\hline & Dec & 2,816 & 2,852 & 9,570 & 10,238 & 2,813 & 3,013 & 1,933 & 2,005 \\
\hline \multirow[t]{12}{*}{2009} & Jan & 2,477 & 2,607 & 8,916 & 9,414 & 2,601 & 2,836 & 1,849 & 1,844 \\
\hline & Feb & 2,380 & 2,365 & 8,426 & 8,879 & 2,389 & 2,451 & 1,674 & 1,688 \\
\hline & Mar & 2,537 & 2,702 & 9,123 & 9,676 & 2,557 & 2,656 & 1,709 & 1,788 \\
\hline & Apr & 2,471 & 2,580 & 8,661 & 9,101 & 2,520 & 2,542 & 1,648 & 1,662 \\
\hline & May & 2,447 & 2,546 & 8,757 & 9,134 & 2,423 & 2,572 & 1,674 & 1,748 \\
\hline & Jun & 2,602 & 2,627 & 8,675 & 9,107 & 2,626 & 2,779 & 1,685 & 1,815 \\
\hline & Jul & 2,742 & 2,893 & 9,390 & 9,726 & 2,840 & 2,955 & 1,926 & 2,012 \\
\hline & Aug & 2,668 & 2,815 & 9,448 & 9,734 & 2,852 & 2,961 & 1,871 & 1,951 \\
\hline & Sep & 2,706 & 2,780 & 9,559 & 9,870 & 2,921 & 2,997 & 1,834 & 1,919 \\
\hline & Oct & 2,535 & 2,680 & 9,270 & 9,673 & 2,789 & 2,787 & 1,713 & 1,887 \\
\hline & Nov & 2,439 & 2,529 & 8,619 & 9,077 & 2,554 & 2,682 & 1,599 & 1,733 \\
\hline & Dec & 2,721 & 2,626 & 9,399 & 9,760 & 2,756 & 2,927 & 1,793 & 1,879 \\
\hline \multirow[t]{12}{*}{2010} & Jan & 2,423 & 2,494 & 8,632 & 9,188 & 2,526 & 2,520 & 1,665 & 1,741 \\
\hline & Feb & 2,250 & 2,367 & 8,039 & 8,367 & 2,263 & 2,392 & 1,514 & 1,539 \\
\hline & Mar & 2,461 & 2,601 & 8,737 & 9,242 & 2,537 & 2,656 & 1,613 & 1,669 \\
\hline & Apr & 2,243 & 2,419 & 8,174 & 8,556 & 2,364 & 2,347 & 1,526 & 1,577 \\
\hline & May & 2,293 & 2,423 & 8,270 & 8,640 & 2,357 & 2,388 & 1,542 & 1,526 \\
\hline & Jun & 2,341 & 2,500 & 8,434 & 8,775 & 2,507 & 2,613 & 1,582 & 1,676 \\
\hline & Jul & 2,613 & 2,640 & 8,433 & 9,004 & 2,603 & 2,755 & 1,670 & 1,758 \\
\hline & Aug & 2,573 & 2,709 & 9,071 & 9,588 & 2,706 & 2,834 & 1,801 & 1,799 \\
\hline & Sep & 2,625 & 2,867 & 9,335 & 9,599 & 2,731 & 2,975 & 1,782 & 1,764 \\
\hline & Oct & 2,484 & 2,550 & 9,206 & 9,605 & 2,566 & 2,786 & 1,609 & 1,711 \\
\hline & Nov & 2,476 & 2,626 & 9,047 & 9,460 & 2,614 & 2,689 & 1,663 & 1,749 \\
\hline & Dec & 2,445 & 2,627 & 9,416 & 9,772 & 2,791 & 2,859 & 1,676 & 1,884 \\
\hline
\end{tabular}


Table 1 (continued)

\begin{tabular}{|c|c|c|c|c|c|c|c|c|c|}
\hline \multicolumn{2}{|l|}{ Date } & \multicolumn{2}{|c|}{ Alabama } & \multicolumn{2}{|l|}{ Florida } & \multicolumn{2}{|c|}{ Louisiana } & \multicolumn{2}{|c|}{ Mississippi } \\
\hline year & month & female & male & female & male & female & male & female & male \\
\hline \multirow[t]{12}{*}{2011} & Jan & 2,385 & 2,564 & 8,626 & 9,112 & 2,410 & 2,620 & 1,594 & 1,688 \\
\hline & Feb & 2,219 & 2,284 & 7,937 & 8,270 & 2,346 & 2,380 & 1,510 & 1,531 \\
\hline & Mar & 2,329 & 2,558 & 8,462 & 8,822 & 2,421 & 2,623 & 1,586 & 1,652 \\
\hline & Apr & 2,064 & 2,319 & 7,947 & 8,398 & 2,250 & 2,391 & 1,487 & 1,506 \\
\hline & May & 2,222 & 2,413 & 8,046 & 8,727 & 2,370 & 2,508 & 1,534 & 1,584 \\
\hline & Jun & 2,521 & 2,597 & 8,445 & 8,838 & 2,526 & 2,532 & 1,556 & 1,676 \\
\hline & Jul & 2,582 & 2,646 & 8,965 & 9,323 & 2,624 & 2,757 & 1,669 & 1,780 \\
\hline & Aug & 2,773 & 2,839 & 9,466 & 9,866 & 2,748 & 3,026 & 1,802 & 1,852 \\
\hline & Sep & 2,465 & 2,662 & 9,293 & 9,898 & 2,709 & 2,808 & 1,779 & 1,729 \\
\hline & Oct & 2,384 & 2,478 & 9,088 & 9,242 & 2,614 & 2,677 & 1,672 & 1,647 \\
\hline & Nov & 2,412 & 2,497 & 8,850 & 9,295 & 2,579 & 2,692 & 1,644 & 1,760 \\
\hline & Dec & 2,541 & 2,600 & 8,932 & 9,566 & 2,653 & 2,624 & 1,808 & 1,814 \\
\hline \multirow[t]{12}{*}{2012} & Jan & 2,374 & 2,436 & 8,569 & 8,886 & 2,546 & 2,582 & 1,648 & 1,592 \\
\hline & Feb & 2,284 & 2,341 & 8,231 & 8,522 & 2,411 & 2,559 & 1,516 & 1,565 \\
\hline & Mar & 2,210 & 2,389 & 8,512 & 8,996 & 2,414 & 2,620 & 1,527 & 1,482 \\
\hline & Apr & 2,234 & 2,252 & 7,944 & 8,441 & 2,230 & 2,404 & 1,405 & 1,477 \\
\hline & May & 2,358 & 2,425 & 8,321 & 8,802 & 2,430 & 2,563 & 1,559 & 1,535 \\
\hline & Jun & 2,390 & 2,514 & 8,382 & 8,614 & 2,393 & 2,609 & 1,571 & 1,622 \\
\hline & Jul & 2,485 & 2,656 & 8,846 & 9,215 & 2,693 & 2,903 & 1,689 & 1,790 \\
\hline & Aug & 2,689 & 2,711 & 9,336 & 9,689 & 2,786 & 2,933 & 1,686 & 1,738 \\
\hline & Sep & 2,428 & 2,495 & 9,003 & 9,664 & 2,691 & 2,814 & 1,601 & 1,654 \\
\hline & Oct & 2,482 & 2,599 & 9,410 & 9,819 & 2,762 & 2,810 & 1,666 & 1,773 \\
\hline & Nov & 2,448 & 2,482 & 8,615 & 9,135 & 2,526 & 2,646 & 1,651 & 1,663 \\
\hline & Dec & 2,390 & 2,376 & 8,865 & 9,331 & 2,576 & 2,741 & 1,573 & 1,686 \\
\hline
\end{tabular}

gistic model for a trend and a jump in 2006 [boys $\mathrm{t}_{\mathrm{t}} \sim$ binomial $\left(\mathrm{LB}_{\mathrm{t}}\right.$, $\left.\pi_{t}\right)$ ], where $t$ is the time in months and LB is live births, has the following form:

$$
\log \text { odds }\left(\pi_{\mathrm{t}}\right)=\mathrm{a}_{0}+\mathrm{a}_{1} \times \mathrm{t}+\mathrm{a}_{2} \times \mathrm{d}_{2006}(\mathrm{t}) .
$$

The dummy variable dAprJun2006 for a peak 8-10 months after Hurricane Katrina has the value 1 in April, May and June 2006, and the value 0 elsewhere. The simple but effective seasonal submodel employed has the form:

$$
\text { season }(\mathrm{t})=\mathrm{a}+\mathrm{b} \times \sin (6.28 \times \mathrm{t}+\mathrm{c})+\mathrm{d} \times \cos (12.57 \times \mathrm{t}+\mathrm{e}) \text {, }
$$

where 'a' to 'e' are appropriately estimated parameters specific for the individual US states or combinations of states.

The analysis in this paper was multivariable. The model used contained 4 main parameters and 5 seasonal nested parameters [season ( $t$ ), formula 2]. The explicit model equation in SAS notation is 'model $\mathrm{m} /$ tot $=\mathrm{t}$ season_t dAprJun2006 rain', with the variables: $\mathrm{m}=$ monthly male births; tot = monthly total births; $\mathrm{t}=$ time in months; season_t $=$ seasonal submodel (formula 2, see above); dAprJun2006 = dummy variable for the time window from April to June 2006; rain = total amount of rain per state.

Data were processed with Microsoft Excel 10, and statistical analyses were carried out using SAS 9.3 (SAS Institute Inc., Cary, N.C., USA). A p value $\leq 0.05$ was taken to represent a statistically significant result.

Birth Sex Ratio and Hurricane Katrina

\section{Results}

Of the total 3,903,660 live births, 1,996,966 (51.16\%) were male and 1,906,694 (48.84\%) were female $(\mathrm{M} / \mathrm{F}=$ 1.0473). Comparison of the overall monthly $\mathrm{M} / \mathrm{F}$ trend in Alabama, Florida, Louisiana and Mississippi combined from 2003 to 2012 disclosed no obvious long-term disturbances after Hurricane Katrina. However, there was a significant seasonal variation $(\mathrm{p}=0.0073)$, which means that the seasonal submodel (equation 2, above) significantly improved the fit of the ordinary linear logistic time trend model. Moreover, 8-10 months (April to June 2006) after the hurricane, M/F jumped significantly from 1.052 to 1.071 with a SOR of 1.018 (95\% CI 1.005, 1.032, p = 0.0074; fig. 2).

The state of Mississippi was hit the hardest by rainfall during the passage of Hurricane Katrina (fig. 1). Comparison of the M/F trends of Florida, which experienced below 10 inches of precipitation during the storm, with Alabama, Louisiana and Mississippi combined, all of which experienced heavy rainfall of more than 10 inches (fig. 1; table 2), disclosed that in Florida no peak from April to June was 
present (SOR 1.002, 95\% CI 0.984, 1.019, $\mathrm{p}=0.8661$; fig. 3), whereas a highly significant peak was evident in Alabama, Louisiana and Mississippi (SOR 1.042, 95\% CI 1.021, 1.064, $\mathrm{p}<0.0001$; fig. 4). The SOR peak 4-6/2006 together with the $95 \%$ confidence intervals for all four individual states by the overall sum of rainfall in these states within the 3 days of the passage of Hurricane Katrina is displayed in figure 5. This monotone relationship suggests a possible causal relation between rain and an increase in $\mathrm{M} / \mathrm{F}$ 8-10 months after exposure. A simple proportion calculation involving the additional M/F increase shown over 3 months in Alabama, Louisiana and Mississippi translated to a deficit of approximately 800 girls if, theoretically, only girls were affected by lethal mutations.

\section{Discussion}

In this study, there was a dose-response relationship between rainfall after Hurricane Katrina and the monthly M/F ratio of live births 8-10 months later. Rainfall generally increases the ambient background radiation, and radiation in turn is known to increase the sex ratio [15, 16]. Stress from any cause tends to lower M/F due to an excess male foetal losses $[6,9,17]$.

This theory accords with the Trivers-Willard hypothesis, which proposes that evolution through natural selection has favoured individuals who are capable of biasing offspring gender in favour of the sex with the best reproductive prospects in accordance with extant periconceptional and gestational conditions [18-20].

It has been shown that Hurricane Katrina caused foetal loss which was dose-related to property damage [8]. Indeed, it has been estimated that the adjusted odds of foetal death were 1.4 and 2.4 times higher, respectively, in parishes suffering $10-50$ and $>50 \%$ wreckage to available housing stock [10]. For every $1 \%$ increase in the destruction of housing stock, the study showed a $1.7 \%$ increase in foetal losses [10]. The study does not support the Trivers-Willard hypothesis since the foetal losses that occurred shortly after Hurricane Katrina appeared to have affected both genders equally. However, an expected seasonal variation was found, as previously reported [11].

This study has shown a monotone relationship (a positive correlation) that could underpin a possible causal relation between rain or the radioactivity contained therein [21], and M/F 8-10 months after exposure. This was present only in the three states (Alabama, Louisiana and Mississippi) most affected by rainfall and floods. The distinct M/F peak could perhaps be ex-
Table 2. Precipitation in inches for Alabama, Florida, Louisiana and Mississippi for the 3 days $(29-31 / 8 / 2005)$ of the passage of Hurricane Katrina

\begin{tabular}{|c|c|c|c|}
\hline Date & $29 / 8$ & $30 / 8$ & $31 / 8$ \\
\hline \multicolumn{4}{|l|}{ Alabama } \\
\hline Count & 6,221 & 8,673 & 3,988 \\
\hline Minimum & 0.0 & 0.2 & 0.0 \\
\hline Maximum & 5.2 & 10.3 & 1.6 \\
\hline Sum & $1,475.8$ & $14,417.9$ & 452.6 \\
\hline Mean & 0.2 & 1.7 & 0.1 \\
\hline \multicolumn{4}{|l|}{ Louisiana } \\
\hline Count & 4,864 & 5,480 & 535 \\
\hline Minimum & 0.0 & 0.0 & 0.0 \\
\hline Maximum & 10.4 & 10.5 & 0.8 \\
\hline Sum & $8,631.2$ & $6,916.0$ & 20.7 \\
\hline Mean & 1.8 & 1.3 & 0.0 \\
\hline \multicolumn{4}{|l|}{ Mississippi } \\
\hline Count & 5,730 & 8,015 & 1,103 \\
\hline Minimum & 0.0 & 0.5 & 0.0 \\
\hline Maximum & 8.2 & 10.3 & 2.1 \\
\hline Sum & $3,821.1$ & $27,155.5$ & 96.9 \\
\hline Mean & 0.7 & 3.4 & 0.1 \\
\hline \multicolumn{4}{|l|}{ Florida } \\
\hline Count & 8,856 & 8,138 & 7,377 \\
\hline Minimum & 0.0 & 0.0 & 0.0 \\
\hline Maximum & 3.0 & 3.9 & 4.1 \\
\hline Sum & $2,920.8$ & $3,234.3$ & $1,918.2$ \\
\hline Mean & 0.3 & 0.4 & 0.3 \\
\hline
\end{tabular}

The count is the number of readings. Minimum, maximum, sum and mean values are given as inches.

plained by increased ionizing radiation exposure during and after the passage of Hurricane Katrina. Florida failed to show an M/F rise and this may be due to the much smaller amount of rainfall experienced in this state following the storm.

Radiation is a unique toxin in that when both genders within a population are equally exposed, an overall increase in $\mathrm{M} / \mathrm{F}$ is produced since more females than males are lost in utero from lethal mutations [15]. This is because irradiated men sire an excess of males [22] and irradiated females give birth to an excess of females [23,24].

These findings are attributed to the hypothesis that if an X-linked recessive lethal gene is induced in a mother's germ cell line by ionizing radiation, it would have no effect on a heterozygous daughter but would be lethal to a hemizygous male zygote. X-linked dominant lethal mutations in mothers would be equally lethal to both genders [23]. X-linked dominant mutations induced in fathers 


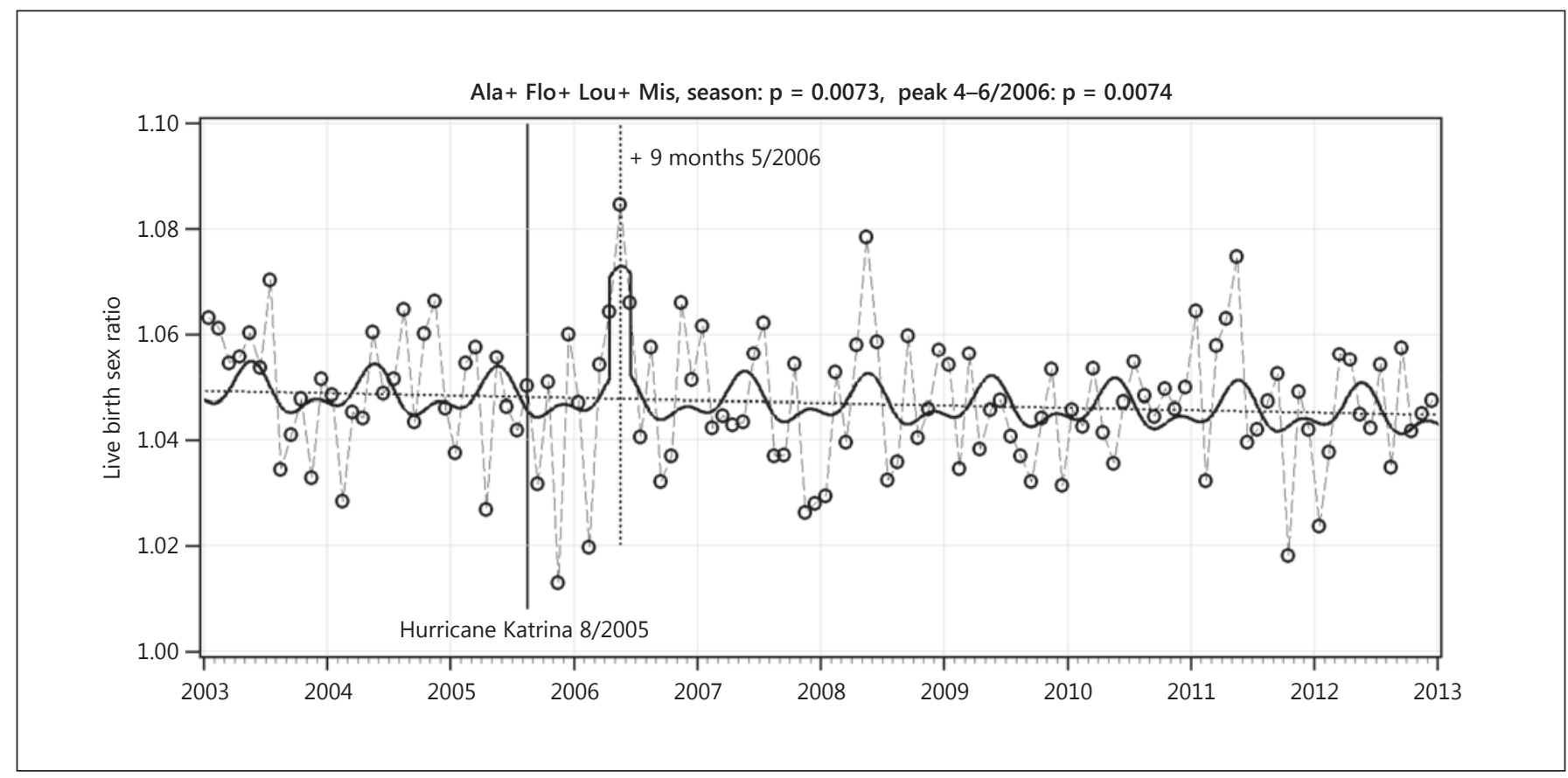

Fig. 2. The M/F trend in Alabama (Ala), Florida (Flo), Louisiana (Lou) and Mississippi (Mis) combined from 2003 to 2012: seasonal ordinary linear logistic regression model (equation 2) with a significant peak between April and June, 2006 (peak 4-6/2006), 8-10 months after Hurricane Katrina.

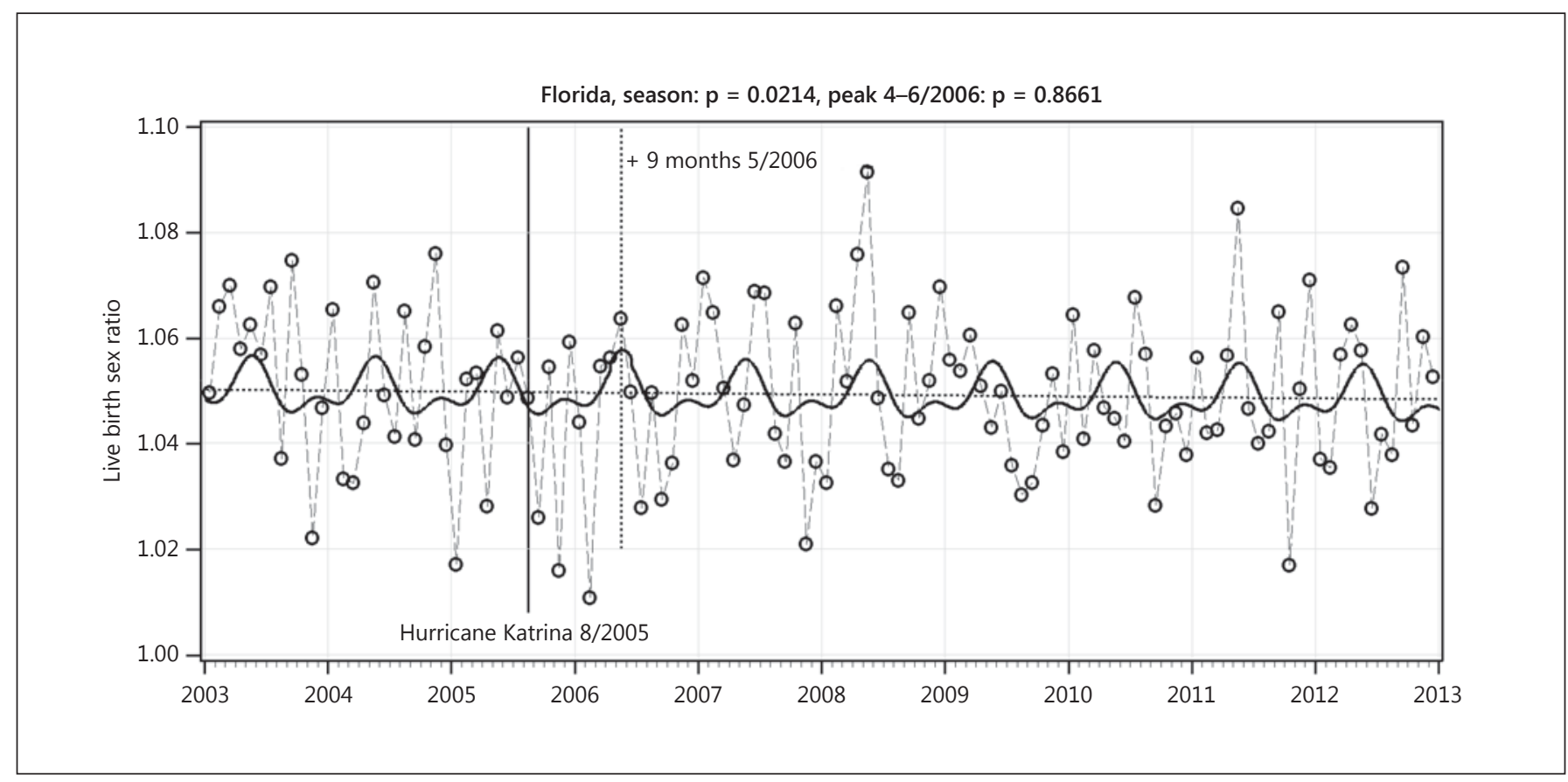

Fig. 3. The M/F trend in Florida from 2003 to 2012: seasonal model (equation 2) with a non-significant peak between April and June, 2006 (peak 4-6/2006), 8-10 months after Hurricane Katrina. 


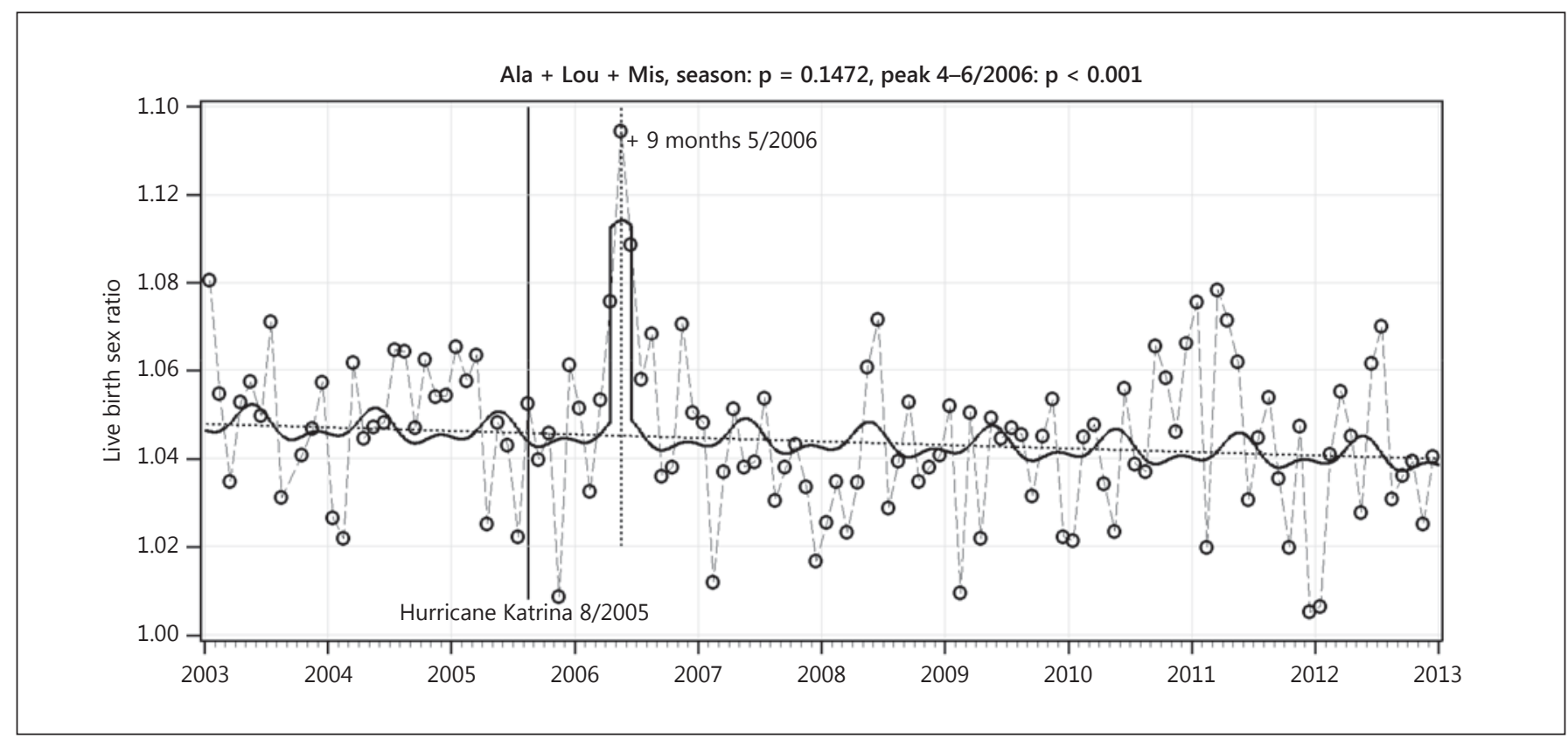

Fig. 4. The M/F trend in Alabama (Ala), Louisiana (Lou) and Mississippi (Mis) combined: seasonal model (equation 2) with a highly significant peak between April and June, 2006 (peak 4-6/2006), 8-10 months after Hurricane Katrina.

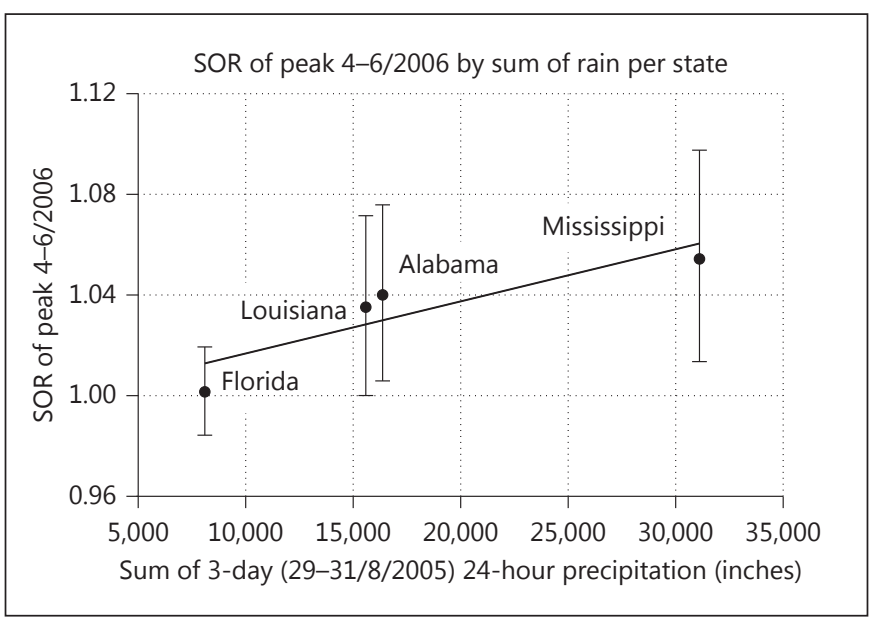

Fig. 5. Monotone association between the peak SOR (including $95 \%$ CIs) between April and June 2006 and total rainfall over Florida, Louisiana, Alabama and Mississippi from August 29 to 31, 2005.

would only suppress female offspring. Recessive X-linked lethal mutations in fathers would not influence the gender ratio as sons do not receive the paternal X chromosome and daughters carry (and are protected by) a second $\mathrm{X}$ chromosome from their mother [23]. The sex ratio is therefore influenced by an increased but gender-biased foetal mortality.
It has been hypothesized that the skew toward higher female mortality may be due to the fact that the X chromosome contains more genetic material and is larger, and hence may be physically more easily struck by ionizing radiation. Another possibility is that ova and sperm afford their genetic material different levels of protection $[16,23]$.

It has long been known that rainfall is positively associated with a rise in $\mathrm{M} / \mathrm{F}$ [12]. It is also known that rainfall is associated with an increase in ambient natural background radiation due to the precipitation of radionuclides [21]. This study appears to confirm this relationship through the induction of a female birth deficit after heavy rainfall.

\section{Conclusion}

This study showed that heavy rainfall and flooding following Hurricane Katrina in the three states most affected were associated with a subsequent rise in $\mathrm{M} / \mathrm{F}$. As rain increases background radiation, and radiation increases $\mathrm{M} / \mathrm{F}$, we are, to the best of our knowledge, the first to suggest that the well-known yet unexplained sex ratio seasonality may be at least partially due to natural or manmade radiation contained in precipitation. 


\section{References}

1 James WH: The human sex ratio. Part 1: a review of the literature. Hum Biol 1987;59:721752.

2 James WH: The human sex ratio. Part 2: a hypothesis and a program of research. Hum Biol 1987;59:873-900.

-3 Lyster WR: Altered sex ratio after the London smog of 1952 and the Brisbane flood of 1965 J Obstet Gynaecol Br Commonw 1974;81: 626-631.

4 Fukuda M, Fukuda K, Shimizu T, et al: Decline in sex ratio at birth after Kobe earthquake. Hum Reprod 1998;13:2321-2322.

5 Zorn B, Sucur V, Stare J, et al: Decline in sex ratio at birth after 10-day war in Slovenia: brief communication. Hum Reprod 2002; 17: 3173-3177.

6 Catalano R, Bruckner T, Marks AR, et al: Exogenous shocks to the human sex ratio: the case of September 11, 2001 in New York City. Hum Reprod 2006;21:3127-3131.

7 Dodla VB, Desamsetti S, Yerramilli A: A comparison of HWRF, ARW and NMM models in Hurricane Katrina (2005) simulation. Int J Environ Res Public Health 2011;8:2447-2469.
8 Darsey DA, Carlton FB Jr, Wilson J: The Mississippi Katrina experience: applying lessons learned to augment daily operations in disaster preparation and management. South Med J 2013;106:109-112.

$\checkmark 9$ Bruckner TA, Catalano R, Ahern J: Male fetal loss in the U.S. following the terrorist attacks of September 11, 2001. BMC Public Health 2010;10:273.

10 Zahran S, Peek L, Snodgrass JG, et al: Economics of disaster risk, social vulnerability, and mental health resilience. Risk Anal 2011; 31:1107-1119.

11 Lerchl A: Seasonality of sex ratio in Germany. Hum Reprod 1998;13:1401-1402.

12 Lyster WR, Bishop MW: An association between rainfall and sex ratio in man. J Reprod Fertil 1965;10:35-47.

13 Centers for Disease Control and Prevention: Vitalstats. http://www.cdc.gov/nchs/vitalstats.htm.

14 NOAA, National Weather Service: Advanced hydrologic prediction service. http://water. weather.gov/precip/download.php.

15 Scherb H, Voigt K: Trends in the human sex odds at birth in Europe and the Chernobyl Nuclear Power Plant accident. Reprod Toxi col 2007;23:593-599.
16 Scherb H, Voigt K: The human sex odds at birth after the atmospheric atomic bomb tests, after Chernobyl, and in the vicinity of nuclear facilities. Environ Sci Pollut Res Int 2011;18:697-707.

17 Catalano RA: Sex ratios in the two Germanies: a test of the economic stress hypothesis. Hum Reprod 2003;18:1972-1975.

18 Trivers RL, Willard DE: Natural selection of parental ability to vary the sex ratio of offspring. Science 1973;179:90-92.

19 Myers JH: Sex ratio adjustment under food stress: maximization of quality or numbers of offspring? Am Naturalist 1978;112:381-388.

20 Williams RJ, Gloster SP: Human sex-ratio as it relates to caloric availability. Soc Biol 1992; 39:285-291.

-21 Livesay RJ, Blessinger CS, Guzzardo TF, et al: Rain-induced increase in background radiation detected by Radiation Portal Monitors. J Environ Radioact 2014;137:137-141.

22 James WH: Ionizing radiation and offspring sex ratio. J Epidemiol Community Health 1997;51:340-341.

23 Vogel F, Motulsky AG: Human Genetics, ed 2. Berlin, Springer, 1986.

24 Schull WJ, Neel JV: Radiation and the sex ratio in man. Science 1958;128:343-348. 